\title{
Clearance of Cystic Duct Remnant Stones Using a SpyGlass Retrieval Basket during Single-Operator Cholangioscopy at ERCP
}

\author{
James Hawken ${ }^{1} \quad$ Jim Portal ${ }^{1}$ \\ ${ }^{1}$ Bristol Liver Unit, University Hospitals Bristol, Bristol, \\ United Kingdom
}

Address for correspondence James Hawken, BM, BS, MRCP, Bristol Liver Unit, University Hospitals Bristol, Marlborough Street, Bristol BS28HW, United Kingdom (e-mail: jameshawken@doctors.org.uk).

J Digest Endosc 2020;11:56-57.

\begin{abstract}
Keywords

- cystic duct remnant stone

- ERCP

- SpyGlass

- SpyGlass retrieval basket

Extraction of stones from a cystic duct remnant following cholecystectomy poses a significant therapeutic dilemma. Surgical intervention has historically been required due to difficulty accessing the cystic duct remnant during conventional endoscopic retrograde cholangiopancreatography. Single-operator cholangioscopy can access the cystic duct remnant, enabling electrohydraulic lithotripsy and stone extraction. Method of stone extraction has seldom been described in this developing field. Clearing duct remnant stones using guidewire placement and an extractor balloon have been reported. This is the first reported case of cystic duct remnant stone extraction using a SpyGlass retrieval basket.
\end{abstract}

\section{Introduction}

Management of cystic duct remnant stones (CDRS) during endoscopic retrograde cholangiopancreatography (ERCP) is challenging. Single-operator cholangioscopy is an emerging modality that enables stone fragmentation and extraction. Clearance of the cystic duct can be difficult, with limited therapeutic techniques available. We describe successful cholangioscopic stone extraction using a SpyGlass retrieval basket.

\section{Case Report}

A 64-year-old patient presented with right upper quadrant pain following cholecystectomy. Ultrasound and magnetic resonance cholangiopancreatography identified stones in the common bile duct and cystic duct remnant. Two ERCPs at the referring hospital were unsuccessful in extracting the CDRS. As pain requiring hospital admissions continued, surgical excision of the cystic duct remnant was considered. Due to high operative risk and patient preference, the

published online January 18,2020
DOI https://doi.org/

$10.1055 / \mathrm{s}-0040-1715282$ ISSN 0976-5042. patient was referred to a tertiary center stone extraction using cholangioscopy. A single-operator system, the SpyGlass Direct Visualization System (Boston Scientific, Natick, Massachusetts, United States), was used. CDRS were fragmented using SpyGlass-guided electrohydraulic lithotripsy (EHL), but it was not possible to retrieve the fragments despite balloon trawl, retrieval net, and dilation of the cystic duct remnant (max $8 \mathrm{~mm}$, Hurricane Biliary Balloon Dilatation Catheter, Boston Scientific). Plastic stents were placed in the cystic and common bile ducts and repeat ERCP was scheduled. During the follow-up procedure, a SpyGlass retrieval basket was deployed through the cholangioscope following stent removal and further EHL. This was successful in retrieving the CDRS, with complete duct clearance achieved. The patient's pain resolved and no stones were seen on follow-up ERCP 1 month later.

\section{Discussion}

CDRS are often removed at the time of laparoscopic cholecystectomy, but this is not an infallible technique. ${ }^{1}$

(C)2020. Society of Gastrointestinal Endoscopy of India.

This is an open access article published by Thieme under the terms of the Creative Commons Attribution-NonDerivative-NonCommercial-License, permitting copying and reproduction so long as the original work is given appropriate credit. Contents may not be used for commercial purposes, or adapted, remixed, transformed or built upon. (https://creativecommons.org/licenses/by-nc-nd/4.0/).

Thieme Medical and Scientific Publishers Pvt. Ltd. A-12, 2nd Floor, Sector 2, Noida-201301 UP, India 
Many patients undergo subtotal cholecystectomy that has a higher incidence of leaving remnant duct stones than conventional laparoscopic cholecystectomy. Incidence of CDRS as the cause of postcholecystectomy syndrome is $16 \% .{ }^{2}$ When CDRS are found in patients presenting with pain after cholecystectomy, stone removal by standard ERCP methods is challenging because of difficulty accessing the duct and due to the variable length of the stump. However, endoscopic management is an attractive proposition with the alternative being open or laparoscopic surgical intervention. Surgical intervention is the established therapeutic option, but often involves a prolonged hospital stay and associated morbidity of $13.3 \%{ }^{3}$

The therapeutic capability of cholangioscopy is increasingly recognized through technical advances and greater availability. However, cystic duct cholangioscopy remains, a branch of this field, still in its infancy. In a multicenter prospective study of 148 patients in Japan who underwent single-operator cholangioscopy, just two were for cystic duct cannulation. Both of these were for gallbladder cytology as access during conventional ERCP failed. ${ }^{4}$

Reports of cholangioscopy for CDRS are very limited, although descriptions of novel techniques are emerging. Sepe et al published a case series on extraction of CDRS using SpyGlass cholangioscopy. Ten of thirteen (76.9\%) patients had complete duct clearance with SpyGlass. ${ }^{1}$ EHL was required to enable clearance in all 10 patients, but the method of stone extraction following EHL was not described. Of the three cases where duct clearance failed, two went on to surgery and one was lost to follow-up. Reports on precise methods of stone extraction from cystic duct remnants following EHL are lacking. Guidewire placement in the cystic duct under direct visualization through a cholangioscope for removal of CDRS was reported in the World Journal of Gastrointestinal Surgery in 2009. ${ }^{5}$ An extractor basket was then passed over the guidewire to extract CDRS. However, such techniques can be difficult as an impacted stone may not allow passage of a guidewire. Successful CDRS clearance using an extractor balloon was reported by Harwani et al in 2016. ${ }^{6}$ Deemed a high-risk surgical candidate due to age, ischemic heart disease, and ejection fraction $<35 \%$, extractor balloon enabled avoidance of high-risk surgery. In such cases, EHL and stone extraction offer an attractive therapeutic option.

\section{Conclusion}

Clearance of CDRS using conventional ERCP techniques is challenging, while surgical approaches carry significant risk in certain patients. Cholangioscopy and EHL can be an extremely useful in managing CDRS, although little has been published on specific methods of stone retrieval of CDRS following EHL. In this patient, stone extraction using a SpyGlass retrieval basket deployed through the cholangioscope was successful where other modalities had failed. This adds to the growing evidence highlighting the capabilities of singleoperator cholangioscopy in managing CDRS.

\section{Learning Points}

- CDRS can pose a significant therapeutic challenge.

- Single-operator cholangioscopy has an emerging role in the management of CDRS.

- A cholangioscopic retrieval basket can be effective for cystic duct stone extraction.

\section{Note}

- Dr Jim Portal carries out consultancy for Boston Scientific. No financial support was provided for this article.

- No prior presentation.

- Both authors conform to the Declaration of Helsinki.

\section{Conflicts of Interest}

Corresponding author has no conflicts of interest. The coauthor carries out consultancy for Boston Scientific.

\section{Acknowledgments}

No financial support or personal assistance received.

\section{References}

1 Sepe PS, Berzin TM, Sanaka S, et al. Single-operator cholangioscopy for the extraction of cystic duct stones (with video) Gastrointest Endosc 2012;75(1):206-210

2 Rozsos I, Magyaródi Z, Orbán P. [Cystic duct syndrome and minimally invasive surgery]. Orv Hetil 1997;138(38):2397-2401

3 Palanivelu C, Rangarajan M, Jategaonkar PA, Madankumar MV, Anand NV. Laparoscopic management of remnant cystic duct calculi: a retrospective study. Ann R Coll Surg Engl 2009;91(1):25-29

4 Kurihara T, Yasuda I, Isayama H, et al. Diagnostic and therapeutic single-operator cholangiopancreatoscopy in biliopancreatic diseases: prospective multicenter study in Japan. World J Gastroenterol 2016;22(5):1891-1901

5 Parsi MA. Peroral cholangioscopy-assisted guidewire placement for removal of impacted stones in the cystic duct remnant. World J Gastrointest Surg 2009;1(1):59-61

6 Harwani Y, Goenka M, Rai V, Goenka U. Cystic duct remnant stone removal by endoscopic retrograde cholangiopancreatography. J Digest Endoscop 2016;7(2):65-67 